\title{
Sex- and Age-Specific Associations of Social Status and Health-Related Behaviors with Health Check Attendance: Findings from the Cross-Sectional Kanazawa Study
}

\author{
Hiromasa Tsujiguchi ${ }^{1}$, Daisuke Hori ${ }^{1}$, Yasuhiro Kambayashi ${ }^{1}$, Toshio Hamagishi ${ }^{1}$, \\ Hiroki Asakura1, Junko Mitoma1, Masami Kitaoka1, Anyenda Enoch Olando1, \\ Nguyen Thi Thu Thao', Yohei Yamada1, Koichiro Hayashi' ${ }^{1}$, Tadashi Konoshita ${ }^{2}$, \\ Takiko Sagara1, Aki Shibata1, Hiroyuki Nakamura1 \\ ${ }^{1}$ Department of Environmental and Preventive Medicine, Graduate School of Medical Sciences, Kanazawa University, \\ Kanazawa, Japan \\ ${ }^{2}$ Third Department of Internal Medicine, Fukui University School of Medicine, Eiheiji, Japan \\ Email: t-hiromasa@med.kanazawa-u.ac.jp
}

How to cite this paper: Tsujiguchi, H., Hori, D., Kambayashi, Y., Hamagishi, T., Asakura, H., Mitoma, J., Kitaoka, M., Olando, A.E., Thao, N.T.T., Yamada, Y., Hayashi, K., Konoshita, T., Sagara, T., Shibata, A. and Nakamura, H. (2017) Sex- and Age-Specific Associations of Social Status and Health-Related Behaviors with Health Check Attendance: Findings from the Cross-Sectional Kanazawa Study. Health, 9 , 1285-1300.

https://doi.org/10.4236/health.2017.99093

Received: May 29, 2017

Accepted: September 3, 2017

Published: September 6, 2017

Copyright $\odot 2017$ by authors and Scientific Research Publishing Inc. This work is licensed under the Creative Commons Attribution International License (CC BY 4.0).

http://creativecommons.org/licenses/by/4.0/

\begin{abstract}
Health checks are key features of primary and secondary disease prevention. The aim of this study was to examine the sex- and age-specific association of social status and health-related behaviors with health check attendance in eligible persons. Data were derived from the Kanazawa Study $2011(\mathrm{n}=12,781)$, a cross-sectional study which investigated all the residents in model areas of Kanazawa City, Ishikawa Prefecture, Japan. We selected participants aged 23 years or older with National Health Insurance $(n=4920)$. Attendance at health checks was the outcome. We used social status and health-related behaviors as predictor variables. We analyzed them by sex and applied stratified analyses by age groups for each sex. The bivariate analyses were conducted by means of cross-tabs. We calculated health check attendance rates by each variable. We used Pearson's $\chi^{2}$-test to examine statistically significant differences. We fitted logistic regression models to estimate adjusted odds ratios (ORs) of attendance in the past one year. We computed ORs in a logistic regression model containing all variables described above. Workingmen and women aged 23 to 39 years and aged 40 to 64 years had significantly increased ORs for health check attendance compared with non-working persons. Men, men aged 23 to 39 years and men aged 65 years or older with more physical activity had significantly increased ORs for health check attendance. Male ex-smokers, female ex- and non-smokers, male ex-smokers aged 65 years or older, and fe-
\end{abstract}


male non-smokers aged 40 to 64 years had significantly increased ORs. The findings suggest that population groups with lower social status or increased risks of adverse health effects are less likely to attend health checks than those with higher social status or decreased risks in particular sex and age groups. It indicates that diverse approaches are required to realize the full benefit of health checks.

\section{Keywords}

Health Check, Social Status, Health-Related Behaviors, Secondary Prevention, Health Care

\section{Introduction}

In view of decreasing lifestyle-related disease rates and increasing life expectancy, primary disease prevention for health promotion and secondary disease prevention for early detection of diseases have become more and more important [1]. The key for such approaches are general and preventive health checks; screenings and interventions which include assessment of an individual's lifestyle risk factors and current health by physical examination and clinical laboratory tests. Despite the importance and benefit of such health checks, their understanding is not sufficient and the attendance rate in Japan is not high enough [2].

The Japanese social security system is based on equality. Health care services are equally offered to everyone. The health insurance system is a combination of the system for employees and their dependents and the community-based system for farmers, self-employed, pensioners and their dependents [3]. The latter system is called National Health Insurance (NHI) and covers almost $30 \%$ of the population in Japan [3]. Health insurers of NHI are obligated to implement health checks by the Japanese National Health Insurance Act and Act on Assurance of Medical Care for Elderly People. These health checks and the followed medical counseling are particularly aimed at decreasing lifestyle-related diseases rates by the early detection of metabolic syndromes and associated factors [4].

Numerous studies investigated the associations between social status and health check attendance [5]-[19]. Lifestyles, which are related to a variety of lifestyle-related diseases [20]-[25], were studied concerning health check attendance in the previous studies [5] [6] [7] [12] [18] [19] [26] [27]. These studies have suggested decreased health check attendance rates among groups with lower social status or unhealthy lifestyles, such as lower income, lower education level, being unemployed, being single, physical inactivity, unhealthy diets, smoking, obesity and poor self-rated health status compared with those groups with higher social status or healthier lifestyles. These findings underline the prevention approach for specific vulnerable groups, although the studies have not reached the generally accepted theory. The success of health checks depends heavily on 
good understanding of changes needed and lifestyle modification after that. It is thus important to understand what affects a person's decision to attend health checks and their motivation to change their lifestyle following the health checks. Even if it is difficult to compare attendance rates at health checks globally because health checks are defined differently in various health care systems by sex and age, understanding the determinants in health check attendance featured by sex and age is important in order to improve the preventive health strategy. To the best of our knowledge, studies on associations of social status and health-related behaviors with health check attendance using one study population in all age groups are very few. Therefore, we aimed to examine the sex- and age-specific association between social status and health-related behaviors, including lifestyle and mental health status, with health check attendance in the model areas of Kanazawa city where we could undertake the complete survey.

\section{Materials and Methods}

\subsection{Data Source}

Data were derived from the cross-sectional Kanazawa Study 2011, which investigated the residents in two model areas of Kanazawa, Ishikawa, Japan. Information on social status, lifestyle, mental health status, and use of health services was gathered in the study.

\subsection{Study Sample}

Questionnaires were distributed to 12,871 people, all residents aged 12 years or older in the model areas in November 2011. A total of 12,253 respondents completed the questionnaire. The response rate was $95.2 \%$. To make us well understand the relationship of social status, health-related behaviors with health check attendance, we excluded participants who are obligated to attend health checks by the Japanese Industrial Safety and Health Act or the Japanese School Health and Safety Act. People with employee's health insurance and students are obligated by these Acts. On the other hand, people with National Health Insurance are not obligated to attend health checks, so they have the choice of whether to attend or not. Exclusion criteria are shown on Figure 1. On these analyses, we included participants with National Health Insurance aged 23 years or older $(\mathrm{n}=$ 4920).

\subsection{Outcome Measures}

The outcome measures of the analyses were self-reported attendances at health checks. Participants were asked whether they had attended the health checks recommended by the National Health Insurers in the past year. We created a binary variable indicating health check attendance in the past year (yes $=1$, no $=0$ ).

\subsection{Predictor Variables}

Age was stratified in three intervals, 1) 23 to 39 years (young), 2) 40 to 64 years 


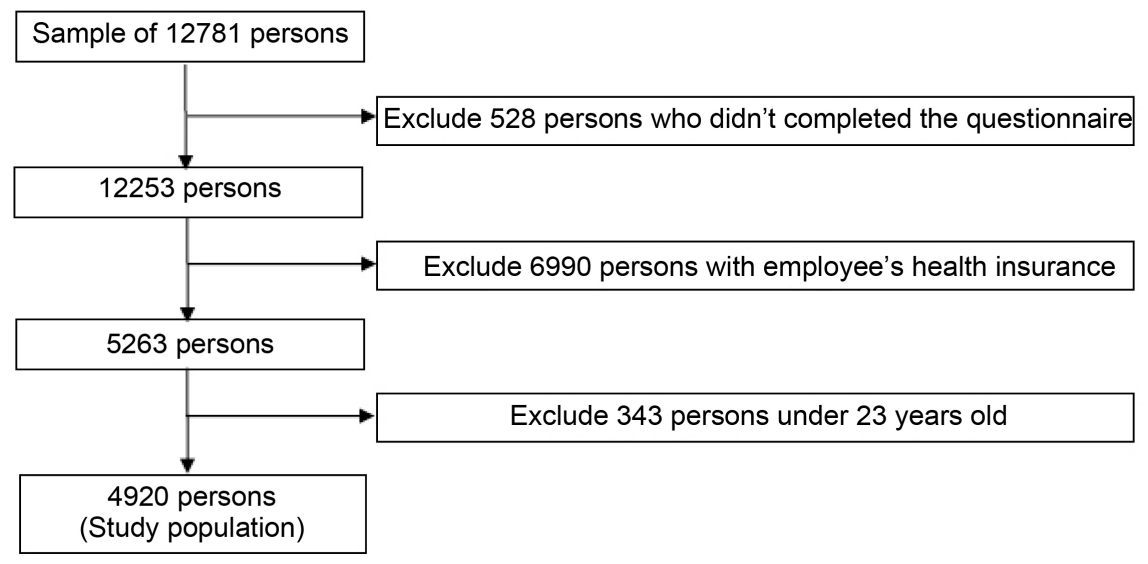

Figure 1. Exclusions from the study sample.

(middle-aged) and 3) 65 years or older (elderly). We used social status and health-related behaviors as predictor variables. A questionnaire was used to ascertain predictor variables. We classified self-rated health status as 1) poor or very poor and 2) very good or good. Working was classified as 1) non-working and 2) working. We categorized household size as 1) one person and 2) more than one person. Physical activity was ascertained by questions on the number of days per week of physical activity including walking and gymnastics. We classified physical activity as 1) not active at all (no weekly physical activity), 2) slightly active (less than 7 days per week), and 3) active (every day). Participants were asked how many days per week they consume vegetables. We categorized the number of days as 1) low (never or 1 to 3 days per week), 2) medium (4 to 6 days per week) and 3) high (everyday). Alcohol consumption was ascertained by questions on the number of days per week when alcohol was consumed. We categorized it as 1) almost every day, 2) sometimes (1 to 5 days per week) and 3) never. We assessed participants smoking status by including a question for participants to categorize themselves as 1) smokers, 2) ex-smokers or 3) nonsmokers. Referring to the World Health Organization (WHO) definition [28], we categorized Body mass index (BMI) in three groups, 1) preobese or obese (25 or over), 2) normal weight (18.5 to 25), 3) underweight (less than 18.5). BMI was calculated using self-reported height and weight. Mental health status was measured using the GHQ-12. The General Health Questionnaire (GHQ) is a self-administered screening questionnaire, designed for use in counseling aimed at detecting individuals with diagnosable psychiatric disorders [29]. In its original version, it had 60 items (GHQ-60), which were reduced to 30 (GHQ-30), 28 (GHQ-28) and 12 items (GHQ-12). The 12-Item General Health Questionnaire is the most usually used screening instrument for common mental disorders, in addition to being a general measure of psychiatric well-being [30]. Numerous studies have assessed the validity of the GHQ for use with adults [31]. Our categories were 1) poor (scored 3 or over), 2) slightly good (scored 1 to 3) and 3) good (scored 0). 


\subsection{Statistical Analysis}

We excluded the bias due to sex or age as much as possible. Firstly, we analyzed the data by sex. After that we applied stratified analyses by age groups for each sex. The bivariate analyses were conducted by means of cross-tabs. We calculated health check attendance rates by each variable. We used Pearson's $\chi^{2}$-test to examine statistically significant differences. We fitted logistic regression models to estimate adjusted odds ratios (ORs) of attendance in the past one year. We computed ORs in logistic regression model containing all variables described above (Working, Household size, Physical activity, Vegetable consumption, Alcohol consumption, Smoking, BMI, Self-rated health status, Mental health). We considered $\mathrm{p}$-value of $<0.05$ to indicate statistical significance; all tests were two-tailed. All statistical analyses were performed using SPSS version 19 for Windows.

\section{Results}

\subsection{Characteristics}

Study participants characteristics are shown on Table 1.

\subsection{Bivariate Analysis}

The one year health check attendance rate was $61.6 \%$ in men and $59.9 \%$ in women. Working, physical activity, vegetable consumption, smoking, self-rated health status, and mental health status was associated with health check attendance in particular sex and age groups (Table 2).

Workingmen had significantly increased health check attendance rate (Working $=63.9 \%, p<0.01)$ compared with non-working persons. However, the significance was not observed in working women. Working men and women aged 23 to 39 years (Working men $=69.9 \%, p<0.001$; Working women $=50.5 \%, \mathrm{p}<$ 0.001 ), and aged 40 to 64 years (Working men $=58.7 \%, \mathrm{p}<0.001$; Working women $=57.3 \%, p<0.05)$ had significantly increased attendance rates. There was no significance in other working persons.

Men with more physical activity had significantly increased attendance rates (Not active at all $=53.9 \%$, Slightly active $=66.2 \%$, Active $=68.9 \%, p<0.001)$, although women with more physical activity did not. Men aged 23 to 39 years (Not active at all $=51.8 \%$, Slightly active $=64.6 \%$, Active $=86.2 \%, \mathrm{p}<0.001)$ and men aged 65 years or older (Not active at all $=59.8 \%$, Slightly active $=66.9 \%$, Active $=86.2 \%, \mathrm{p}<0.05)$ with more physical activity had significantly increased attendance rates, although persons in other groups with that did not.

Women who consumed more vegetables had significantly increased attendance rates $($ Low $=49.7 \%$, Medium $=52.9 \%$, High $=62.1 \%, p<0.001)$, although men with more vegetable consumption did not. Women aged 40 to 64 years (Low $=43.8 \%$, Medium $=44.4 \%$, High $=59.3 \%, \mathrm{p}<0.001)$ and women aged 65 years or older $($ Low $=56.4 \%$, Medium $=60.0 \%$, High $=67.4 \%, p<0.05)$ with more vegetable consumption had significantly increased attendance rates. 
Table 1. Characteristics of the participants with national health insurance aged 23 years or older.

\begin{tabular}{|c|c|c|c|c|c|c|c|c|c|c|c|c|c|c|c|c|}
\hline & \multicolumn{8}{|c|}{ Men } & \multicolumn{8}{|c|}{ Women } \\
\hline & \multicolumn{2}{|c|}{$\begin{array}{c}\text { Total } \\
(\mathrm{n}=2137)\end{array}$} & \multicolumn{2}{|c|}{$\begin{array}{c}23 \text { - } 39 \text { years } \\
(n=318)\end{array}$} & \multicolumn{2}{|c|}{$\begin{array}{c}40-64 \text { years } \\
(n=710)\end{array}$} & \multicolumn{2}{|c|}{$\begin{array}{l}65+\text { years } \\
(\mathrm{n}=1109)\end{array}$} & \multicolumn{2}{|c|}{$\begin{array}{c}\text { Total } \\
(\mathrm{n}=2783)\end{array}$} & \multicolumn{2}{|c|}{$\begin{array}{c}23 \text { - } 39 \text { years } \\
(n=377)\end{array}$} & \multicolumn{2}{|c|}{$\begin{array}{c}40-64 \text { years } \\
(n=918)\end{array}$} & \multicolumn{2}{|c|}{$\begin{array}{l}65+\text { years } \\
(n=1488)\end{array}$} \\
\hline & $\mathbf{n}$ & $\%$ & $\mathrm{n}$ & $\%$ & $\mathbf{n}$ & $\%$ & $\mathbf{n}$ & $\%$ & $\mathbf{n}$ & $\%$ & $\mathbf{n}$ & $\%$ & $\mathbf{n}$ & $\%$ & $\mathbf{n}$ & $\%$ \\
\hline \multicolumn{17}{|l|}{ Working } \\
\hline Non-working & 1015 & 47.9 & 39 & 12.4 & 133 & 18.9 & 843 & 76.5 & 777 & 28.3 & 39 & 10.5 & 83 & 9.1 & 655 & 44.7 \\
\hline Working & 1106 & 52.1 & 276 & 87.6 & 571 & 81.1 & 259 & 23.5 & 1972 & 71.7 & 334 & 89.5 & 828 & 90.9 & 810 & 55.3 \\
\hline \multicolumn{17}{|l|}{ Household size } \\
\hline One person & 99 & 4.6 & 14 & 4.4 & 35 & 4.9 & 50 & 4.5 & 127 & 4.6 & 24 & 6.4 & 37 & 4.0 & 66 & 4.4 \\
\hline More than one person & 2038 & 95.4 & 304 & 95.6 & 675 & 95.1 & 1059 & 95.5 & 2656 & 95.4 & 353 & 93.6 & 881 & 96.0 & 1422 & 95.6 \\
\hline \multicolumn{17}{|l|}{ Physical activity } \\
\hline Not active at all & 850 & 41.5 & 143 & 45.8 & 332 & 48.2 & 375 & 35.7 & 1227 & 46.8 & 231 & 62.3 & 461 & 51.2 & 535 & 39.6 \\
\hline Slightly active & 736 & 35.9 & 102 & 32.7 & 218 & 31.6 & 416 & 39.7 & 891 & 34.0 & 98 & 26.4 & 281 & 31.2 & 512 & 37.9 \\
\hline Active & 464 & 22.6 & 67 & 21.5 & 139 & 20.2 & 258 & 24.6 & 504 & 19.2 & 42 & 11.3 & 159 & 17.6 & 303 & 22.4 \\
\hline \multicolumn{17}{|l|}{ Vegetable consumption } \\
\hline Low & 455 & 21.7 & 74 & 23.8 & 181 & 26.0 & 200 & 18.5 & 329 & 12.1 & 66 & 17.6 & 110 & 12.1 & 153 & 10.6 \\
\hline Medium & 357 & 17.1 & 87 & 28.0 & 134 & 19.2 & 136 & 12.5 & 274 & 10.0 & 59 & 15.7 & 93 & 10.2 & 122 & 8.4 \\
\hline High & 1280 & 61.2 & 150 & 48.2 & 382 & 54.8 & 748 & 69.0 & 2126 & 77.9 & 250 & 66.7 & 706 & 77.7 & 1170 & 81.0 \\
\hline \multicolumn{17}{|l|}{ Alcohol consumption } \\
\hline Everyday & 858 & 42.0 & 73 & 23.7 & 319 & 46.6 & 466 & 44.3 & 296 & 11.5 & 35 & 9.5 & 169 & 18.9 & 92 & 7.0 \\
\hline Sometimes & 558 & 27.3 & 127 & 41.2 & 192 & 28.0 & 239 & 22.7 & 666 & 25.8 & 155 & 41.9 & 286 & 32.0 & 225 & 17.0 \\
\hline Never & 629 & 30.8 & 108 & 35.1 & 174 & 25.4 & 347 & 33.0 & 1622 & 62.8 & 180 & 48.6 & 439 & 49.1 & 1003 & 76.0 \\
\hline \multicolumn{17}{|l|}{ Smoking } \\
\hline Smoker & 624 & 30.5 & 137 & 44.6 & 285 & 41.7 & 202 & 19.1 & 255 & 9.8 & 69 & 18.6 & 122 & 13.7 & 64 & 4.8 \\
\hline Ex-smoker & 941 & 45.9 & 59 & 19.2 & 267 & 39.0 & 615 & 58.2 & 328 & 12.6 & 70 & 18.9 & 144 & 16.2 & 114 & 8.5 \\
\hline Non-smoker & 483 & 23.6 & 111 & 36.2 & 132 & 19.3 & 240 & 22.7 & 2017 & 77.6 & 232 & 62.5 & 624 & 70.1 & 1161 & 86.7 \\
\hline \multicolumn{17}{|l|}{ BMI } \\
\hline Preobese/obese & 467 & 22.7 & 61 & 20.1 & 203 & 29.3 & 203 & 19.1 & 411 & 15.9 & 31 & 9.0 & 129 & 15.0 & 251 & 18.2 \\
\hline Normal weight & 1488 & 72.3 & 229 & 75.3 & 468 & 67.6 & 791 & 74.5 & 1840 & 71.3 & 248 & 72.3 & 624 & 72.4 & 968 & 70.3 \\
\hline Underweight & 103 & 5.0 & 14 & 4.6 & 21 & 3.0 & 68 & 6.4 & 331 & 12.8 & 64 & 18.7 & 109 & 12.6 & 158 & 11.5 \\
\hline \multicolumn{17}{|l|}{ Self-rated health status } \\
\hline Poor/very poor & 566 & 27.4 & 46 & 14.8 & 160 & 23.0 & 360 & 34.0 & 748 & 27.8 & 45 & 12.2 & 169 & 18.6 & 534 & 37.8 \\
\hline Very good/good & 1499 & 72.6 & 264 & 85.2 & 536 & 77.0 & 699 & 66.0 & 1920 & 72.2 & 324 & 87.8 & 738 & 81.4 & 878 & 62.2 \\
\hline \multicolumn{17}{|l|}{ Mental health } \\
\hline Poor & 604 & 29.3 & 109 & 34.9 & 212 & 30.7 & 283 & 26.8 & 1032 & 39.0 & 178 & 47.7 & 352 & 39.0 & 502 & 36.6 \\
\hline Slightly good & 476 & 23.1 & 72 & 23.1 & 174 & 25.2 & 230 & 21.8 & 634 & 24.0 & 87 & 23.3 & 228 & 25.3 & 319 & 23.3 \\
\hline Good & 978 & 47.5 & 131 & 42.0 & 305 & 44.1 & 542 & 51.4 & 981 & 37.1 & 108 & 29.0 & 322 & 35.7 & 551 & 40.2 \\
\hline
\end{tabular}

Women who consumed less alcohol had significantly increased attendance rates $($ Everyday $=52.8 \%$, Sometimes $=58.3 \%$, Never $=60.6 \%, \mathrm{p}<0.05)$.

Femalenon-smoker had significantly increased attendance rates $($ Smoker $=$ 
Table 2. Health check attendance rates in the past one year by variables.

(a)

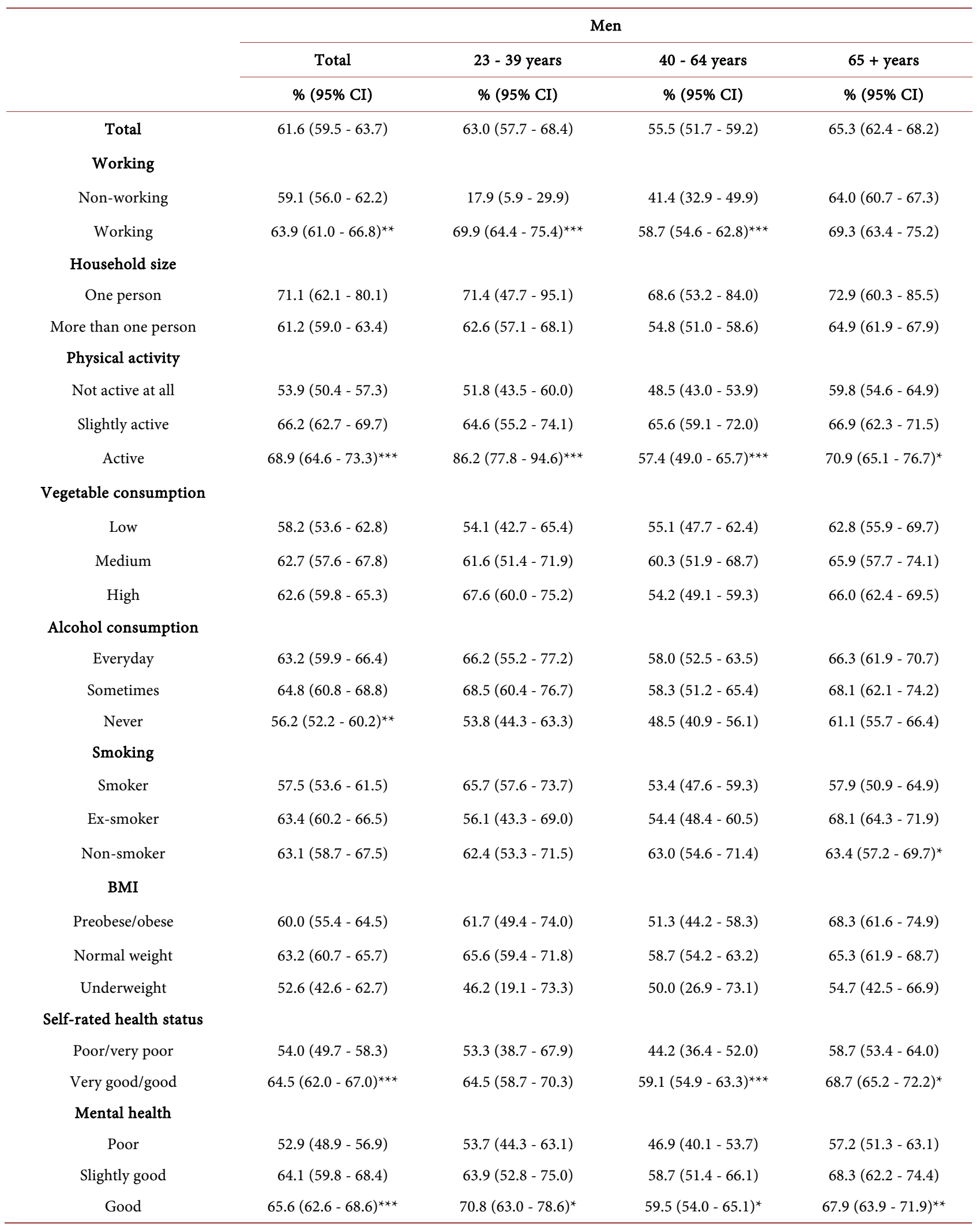

$\mathrm{CI}=$ confidence interval. ${ }^{*} \mathrm{p}<0.05 .{ }^{* *} \mathrm{p}<0.01 .{ }^{* * *} \mathrm{p}<0.001$. 
(b)

\begin{tabular}{|c|c|c|c|}
\hline \multicolumn{4}{|c|}{ Women } \\
\hline Total & 23 - 39 years & $40-64$ years & $65+$ years \\
\hline$\%(95 \% \mathrm{CI})$ & $\%(95 \% \mathrm{CI})$ & $\%(95 \% \mathrm{CI})$ & $\%(95 \% \mathrm{CI})$ \\
\hline $59.9(58.0-61.8)$ & $47.3(42.2-52.4)$ & $55.8(52.5-59.1)$ & $66.0(63.4-68.5)$ \\
\hline $59.9(56.3-63.5)$ & $20.5(7.8-33.2)$ & $42.5(31.7-53.3)$ & $64.9(61.0-68.8)$ \\
\hline $59.8(57.6-62.0)$ & $50.5(45.1-55.9)^{\star * \star}$ & $57.3(53.9-60.7)^{*}$ & $66.5(63.1-69.9)$ \\
\hline $58.3(49.5-67.1)$ & $37.5(18.1-56.9)$ & $55.6(39.4-71.8)$ & $68.3(56.5-80.1)$ \\
\hline $60.0(58.1-61.9)$ & $48.0(42.7-53.3)$ & $55.8(52.5-59.1)$ & $65.8(63.2-68.4)$ \\
\hline $54.1(51.2-56.9)$ & $49.1(42.6-55.6)$ & $52.8(48.2-57.4)$ & $57.4(53.1-61.8)$ \\
\hline $64.6(61.4-67.9)$ & $43.3(33.4-53.2)$ & $62.8(57.1-68.6)$ & $70.1(65.9-74.2)$ \\
\hline $63.2(58.8-67.5)^{\star * *}$ & $48.8(33.5-64.1)$ & $54.2(46.4-62.1)^{\star}$ & $70.1(64.8-75.5)^{\star * *}$ \\
\hline $49.7(44.1-55.3)$ & $45.5(33.4-57.5)$ & $43.8(34.3-53.3)$ & $56.4(48.0-64.8)$ \\
\hline $52.9(46.8-58.9)$ & $51.8(38.7-64.9)$ & $44.4(34.2-54.7)$ & $60.0(51.0-69.0)$ \\
\hline $62.1(60.0-64.2)^{\star * *}$ & $46.7(40.5-53.0)$ & $59.3(55.6-63.0)^{\star * *}$ & $67.4(64.6-70.2)^{\star}$ \\
\hline $52.8(47.0-58.6)$ & $36.4(20.0-52.8)$ & $51.2(43.6-58.9)$ & $61.8(51.7-71.9)$ \\
\hline $58.3(54.4-62.1)$ & $49.3(41.4-57.3)$ & $55.8(49.9-61.7)$ & $68.1(61.8-74.5)$ \\
\hline $60.6(58.1-63.0)^{\star}$ & $46.1(38.7-53.4)$ & $57.8(53.1-62.5)$ & $64.6(61.5-67.7)$ \\
\hline $42.4(36.3-48.6)$ & $36.4(24.8-48.0)$ & $38.3(29.6-47.0)$ & $57.6(45.0-70.2)$ \\
\hline $55.4(49.9-60.9)$ & $42.6(30.9-54.4)$ & $55.0(46.8-63.2)$ & $64.2(55.0-73.3)$ \\
\hline $62.2(60.0-64.4)^{* * *}$ & $50.9(44.4-57.3)$ & $59.7(55.7-63.6)^{\star * *}$ & $66.1(63.3-68.9)$ \\
\hline $60.2(55.3-65.1)$ & $51.7(33.5-69.9)$ & $57.4(48.6-66.2)$ & $62.8(56.5-69.0)$ \\
\hline $60.5(58.2-62.8)$ & $47.8(41.5-54.0)$ & $56.0(52.0-59.9)$ & $67.0(64.0-70.1)$ \\
\hline $57.7(52.2-63.2)$ & $46.8(34.4-59.2)$ & $59.8(50.5-69.1)$ & $61.0(52.9-69.0)$ \\
\hline $58.1(54.4-61.8)$ & $48.9(34.3-63.5)$ & $50.6(42.9-58.3)$ & $61.5(57.1-65.9)$ \\
\hline $60.3(58.1-62.5)$ & $47.0(41.5-52.5)$ & $57.0(53.4-60.6)$ & $68.3(65.1-71.5)^{\star}$ \\
\hline $55.5(52.4-58.6)$ & $45.5(38.1-52.8)$ & $50.4(45.2-55.7)$ & $62.9(58.6-67.3)$ \\
\hline $61.8(57.9-65.6)$ & $54.0(43.6-64.5)$ & $57.4(50.8-64.0)$ & $67.1(61.8-72.4)$ \\
\hline $62.5(59.4-65.6)^{\star *}$ & $44.9(35.4-54.3)$ & $60.4(55.0-65.8)^{*}$ & $67.4(63.4-71.4)$ \\
\hline
\end{tabular}

42.4\%, Non-smoker $=62.2 \%, \mathrm{p}<0.001)$. Male ex-smoker aged 65 years or older $($ Smoker $=57.9 \%$, Ex-smoker $=68.1 \%, \mathrm{p}<0.05)$ and female non-smoker aged 40 to 64 years $($ Smoker $=38.3 \%$, Non-smoker $=59.7 \%, \mathrm{p}<0.001)$ had significantly increased attendance rates.

Men with better self-rated health status had significantly increased attendance rate $($ Very good/good $=64.5 \%, \mathrm{p}<0.001)$. Men aged 40 to 64 years (Very good/ good $=59.1 \%, \mathrm{p}<0.001)$, men aged 65 years or older $($ Very good $/$ good $=68.7 \%$, $\mathrm{p}<0.05)$, and women aged 65 years or older (Very good/good $=68.3 \%, \mathrm{p}<0.05)$ with that had significantly increased attendance rates.

Men $($ Poor $=52.9 \%$, Slightly good $=64.1 \%$, Good $=65.6 \%, p<0.001)$ and women $($ Poor $=55.5 \%$, Slightly good $=61.8 \%$, Good $=62.5 \%, \mathrm{p}<0.01)$ with better mental health had significantly increased attendance rates. Men aged 23 to 
39 years $($ Poor $=53.7 \%$, Slightly good $=63.9 \%$, Good $=70.8 \%, \mathrm{p}<0.05)$, men aged 40 to 64 years $($ Poor $=46.9 \%$, Slightly good $=58.7 \%$, Good $=59.5 \%, \mathrm{p}<$ 0.05 ) and women aged 40 to 64 years (Poor $=50.4 \%$, Slightly good $=57.4 \%$, Good $=60.4 \%, \mathrm{p}<0.05)$ with better mental health had significantly increased attendance rates.

\subsection{Multivariate Logistic Regression Analysis}

Compared with bivariate analysis, fewer variables were associated with health check attendance. After multivariate adjustment for all variables (Table 3), working, physical activity, smoking and self-rated health were significantly associated with ORs of health check attendance in particular sex and age groups.

Working men and women aged 23 to 39 years (Working men $=6.54, \mathrm{p}<$ 0.001 ; Working women $=3.68, \mathrm{p}<0.01$ ) and aged $40-64$ years $($ Working men $=$ 1.87, $\mathrm{p}<0.05$; Working women $=1.71, \mathrm{p}<0.05$ ) had significantly increased OR for health check attendance, although other workingpersons did not.

Men who undertook more physical activity had significantly increased OR for health check attendance (Slightly active $=1.56, \mathrm{p}<0.001$; Active $=1.71, \mathrm{p}<$ 0.001 ), although women who undertook that did not. Men aged 23 to 39 years (Active $=4.00, \mathrm{p}<0.01$ ), and men aged 65 years or older $($ Active $=1.53, \mathrm{p}<$ $0.05)$ with more physical activity had significantly increased ORs.

Male ex-smoker $($ Ex-smoker $=1.27, \mathrm{p}<0.05)$ and ex- and female non- smoker had significantly increased ORs (Ex-smoker $=1.72, \mathrm{p}<0.01$; Non-smoker $=$ 2.12, $\mathrm{p}<0.001)$. Male ex-smoker aged 65 years or older $($ Ex-smoker $=1.56, \mathrm{p}<$ 0.05 ), and female non-smoker aged 40 to 64 years $($ Non-smoker $=2.15, \mathrm{p}<$ 0.001) had significantly increased ORs.

Concerning about self-rated health status, it was related to health check attendance rate only in women aged 65 years or older (Very good/good $=1.38, \mathrm{p}<$ $0.05)$.

\section{Discussion}

\subsection{Key Findings and Comparison with Other Studies}

The aim of the present study was to examine the sex- and age-specific associations between health-related behaviors and health check attendance in persons eligible for health checks. Non-attenders had a greater proportion of lower social status or health-related risk factors than attenders. In addition, we found sexand age-specific social status or health-related risk factors in association with health check attendance.

A literature review showed that non-attenders have lower social status or less favorable risk factor profile than attenders [27]. Our findings are, in part, consistent with previous studies on the association between those and health check attendance. These studies present a confusing picture about the relationship, portraying inconsistent findings. This may be as a result of differences in the availability of these services among countries. In addition, these studies targeted 
Table 3. Odds ratios of health check attendance in the past one year by variables.

(a)

\begin{tabular}{|c|c|c|c|c|}
\hline & \multicolumn{4}{|c|}{ Men } \\
\hline & Total & 23 - 39 years & $40-64$ years & $65+$ years \\
\hline & Adjusted OR (95\% CI) & Adjusted OR (95\% CI) & Adjusted OR (95\% CI) & Adjusted OR (95\% CI) \\
\hline \multicolumn{5}{|l|}{ Working } \\
\hline Non-working & 1.00 (Ref) & 1.00 (Ref) & 1.00 (Ref) & 1.00 (Ref) \\
\hline Working & $1.20(0.98-1.49)$ & $6.54(2.40-17.84)^{\star * \star}$ & $1.87(1.21-2.90)^{\star}$ & $1.11(0.78-1.58)$ \\
\hline \multicolumn{5}{|l|}{ Household size } \\
\hline One person & 1.00 (Ref) & 1.00 (Ref) & 1.00 (Ref) & 1.00 (Ref) \\
\hline More than one person & $0.61(0.37-1.00)$ & $0.70(0.17-2.94)$ & $0.55(0.24-1.23)$ & $0.78(0.38-1.58)$ \\
\hline \multicolumn{5}{|l|}{ Physical activity } \\
\hline Not active at all & 1.00 (Ref) & 1.00 (Ref) & 1.00 (Ref) & 1.00 (Ref) \\
\hline Slightly active & $1.56(1.24-1.96)^{\star * *}$ & $1.33(0.71-2.47)$ & $2.14(1.43-3.19)^{\star * *}$ & $1.24(0.89-1.73)$ \\
\hline Active & $1.71(1.31-2.23)^{\star * *}$ & $4.00(1.67-9.58)^{\star \star}$ & $1.26(0.81-1.95)$ & $1.53(1.04-2.27)^{\star}$ \\
\hline \multicolumn{5}{|l|}{ Vegetable consumption } \\
\hline Low & 1.00 (Ref) & 1.00 (Ref) & 1.00 (Ref) & 1.00 (Ref) \\
\hline Medium & $1.08(0.78-1.48)$ & $1.43(0.67-3.06)$ & $1.15(0.69-1.91)$ & $0.84(0.50-1.41)$ \\
\hline High & $1.02(0.80-1.31)$ & $1.36(0.68-2.73)$ & $0.85(0.56-1.28)$ & $0.95(0.65-1.39)$ \\
\hline \multicolumn{5}{|l|}{ Alcohol consumption } \\
\hline Everyday & 1.00 (Ref) & 1.00 (Ref) & 1.00 (Ref) & 1.00 (Ref) \\
\hline Sometimes & $1.12(0.87-1.43)$ & $1.26(0.61-2.60)$ & $0.91(0.61-1.37)$ & $1.25(0.85-1.84)$ \\
\hline Never & $0.90(0.70-1.14)$ & $1.21(0.55-2.65)$ & $0.79(0.51-1.22)$ & $0.92(0.66-1.28)$ \\
\hline \multicolumn{5}{|l|}{ Smoking } \\
\hline Smoker & 1.00 (Ref) & 1.00 (Ref) & 1.00 (Ref) & 1.00 (Ref) \\
\hline Ex-smoker & $1.27(1.01-1.61)^{*}$ & $1.00(0.46-2.18)$ & $0.93(0.64-1.34)$ & $1.56(1.08-2.26)^{\star}$ \\
\hline Non-smoker & $1.25(0.95-1.65)$ & $1.12(0.63-2.30)$ & $1.59(0.97-2.62)$ & $1.15(0.74-1.79)$ \\
\hline \multicolumn{5}{|l|}{ BMI } \\
\hline Preobese/obese & 1.00 (Ref) & 1.00 (Ref) & 1.00 (Ref) & 1.00 (Ref) \\
\hline Normal weight & $1.10(0.87-1.39)$ & $1.00(0.46-1.95)$ & $1.26(0.87-1.83)$ & $0.86(0.59-1.25)$ \\
\hline Underweight & $0.78(0.47-1.30)$ & $0.83(0.19-3.69)$ & $0.81(0.25-2.60)$ & $0.60(0.31-1.16)$ \\
\hline \multicolumn{5}{|l|}{ Self-rated health status } \\
\hline Poor/very poor & 1.00 (Ref) & 1.00 (Ref) & 1.00 (Ref) & 1.00 (Ref) \\
\hline Very good/good & $1.23(0.97-1.56)$ & $1.10(0.51-2.40)$ & $1.34(0.88-2.03)$ & $1.35(0.97-1.88)$ \\
\hline \multicolumn{5}{|l|}{ Mental health } \\
\hline Poor & 1.00 (Ref) & 1.00 (Ref) & 1.00 (Ref) & $1.00(\operatorname{Ref})$ \\
\hline Slightly good & $1.47(1.11-1.94)^{\star *}$ & $1.24(0.60-2.55)$ & $1.44(0.91-2.27)$ & $1.34(0.88-2.04)$ \\
\hline Good & $1.45(1.14-1.84)^{* * *}$ & $1.83(0.95-3.54)$ & $1.45(0.96-2.18)$ & $1.21(0.85-1.73)$ \\
\hline
\end{tabular}

$\mathrm{OR}=$ odds ratio. $\mathrm{CI}=$ confidence interval. $\mathrm{Ref}=$ reference category. ${ }^{*} \mathrm{p}<0.05 .{ }^{* *} \mathrm{p}<0.01 .{ }^{* *} \mathrm{p}<0.001$. Adjusted variables: Working, Household size, Physical activity, Vegetable consumption, Alcohol consumption, Smoking, BMI, Self-rated health status, Mental health. 
(b)

\begin{tabular}{|c|c|c|c|}
\hline \multicolumn{4}{|c|}{ Women } \\
\hline Total & 23 - 39 years & $40-64$ years & $65+$ years \\
\hline Adjusted OR (95\% CI) & Adjusted OR (95\% CI) & Adjusted OR (95\% CI) & Adjusted OR ( $95 \% \mathrm{CI})$ \\
\hline 1.00 (Ref) & 1.00 (Ref) & 1.00 (Ref) & 1.00 (Ref) \\
\hline $1.09(0.88-1.34)$ & $3.68(1.58-8.59)^{\star *}$ & $1.71(1.01-2.89)^{*}$ & $1.01(0.77-1.33)$ \\
\hline 1.00 (Ref) & 1.00 (Ref) & 1.00 (Ref) & 1.00 (Ref) \\
\hline $1.15(0.76-1.73)$ & $1.75(0.68-4.54)$ & $1.17(0.56-2.44)$ & $0.90(0.48-1.68)$ \\
\hline 1.00 (Ref) & 1.00 (Ref) & 1.00 (Ref) & 1.00 (Ref) \\
\hline $1.49(1.22-1.83)^{\star * *}$ & $0.76(0.45-1.29)$ & $1.53(1.09-2.14)^{*}$ & $1.66(1.22-2.25)^{\star \star}$ \\
\hline $1.40(1.09-1.80)^{\star \star}$ & $0.92(0.41-2.03)$ & $1.03(0.69-1.55)$ & $1.61(1.12-2.33)^{*}$ \\
\hline 1.00 (Ref) & 1.00 (Ref) & 1.00 (Ref) & 1.00 (Ref) \\
\hline $0.90(0.62-1.31)$ & $1.16(0.52-2.56)$ & $0.78(0.41-1.47)$ & $0.90(0.49-1.63)$ \\
\hline $1.27(0.94-1.66)$ & $0.96(0.51-1.78)$ & $1.42(0.87-2.31)$ & $1.23(0.78-1.94)$ \\
\hline 1.00 (Ref) & 1.00 (Ref) & 1.00 (Ref) & 1.00 (Ref) \\
\hline $1.07(0.79-1.46)$ & $1.69(0.70-4.09)$ & $1.00(0.65-1.54)$ & $1.28(0.71-2.28)$ \\
\hline $1.12(0.84-1.49)$ & $1.16(0.48-2.80)$ & $1.09(0.72-1.64)$ & $1.21(0.73-2.02)$ \\
\hline 1.00 (Ref) & 1.00 (Ref) & 1.00 (Ref) & 1.00 (Ref) \\
\hline $1.72(1.18-2.49)^{\star \star}$ & $1.59(0.70-3.60)$ & $1.62(0.95-2.78)$ & $1.72(0.81-3.67)$ \\
\hline $2.12(1.56-2.88)^{* \star *}$ & $1.83(0.93-3.60)$ & $2.15(1.37-3.36)^{* *}$ & $1.65(0.89-3.07)$ \\
\hline 1.00 (Ref) & 1.00 (Ref) & 1.00 (Ref) & 1.00 (Ref) \\
\hline $0.98(0.76-1.26)$ & $0.77(0.33-1.80)$ & $0.96(0.63-1.47)$ & $1.08(0.76-1.54)$ \\
\hline $1.06(0.75-1.50)$ & $0.71(0.27-1.88)$ & $1.44(0.80-2.57)$ & $1.09(0.65-1.82)$ \\
\hline 1.00 (Ref) & 1.00 (Ref) & 1.00 (Ref) & 1.00 (Ref) \\
\hline $1.13(0.91-1.40)$ & $0.81(0.38-1.74)$ & $1.19(0.80-1.76)$ & $1.38(1.03-1.86)^{\star}$ \\
\hline 1.00 (Ref) & 1.00 (Ref) & 1.00 (Ref) & 1.00 (Ref) \\
\hline $1.11(0.88-1.41)$ & $1.25(0.69-2.27)$ & $1.15(0.79-1.69)$ & $1.04(0.72-1.48)$ \\
\hline $1.10(0.89-1.37)$ & $0.96(0.55-1.67)$ & $1.08(0.76-1.53)$ & $1.04(0.75-1.44)$ \\
\hline
\end{tabular}

different age groups and analyzed without stratifying by sex and age groups.

Previous studies showed decreased health check attendance rate was associated with being unemployed [13] [15]. We found decreased health check attendance rate was associated with being non-working particularly in younger and elderly persons. In these age groups, social status may have effect on the decision of attending health checks.

A preceding study in Germany [18] which targeted people aged 35 years or older and analyzed by sex showed that people who were more physically active were likely to attend health checks. Our study also showed that men with more physical activity are likely to attend. In addition to these findings, we found that men in the young age group and men in the elderly age group with more physical activity were likely to attend. Persons with more physical activity in specific sex and age groups had a positive relationship with health check attendance. One 
possible explanation is that a person who exercises daily may be more willing to spare time for health checks, whereas person without any interest in their own health may give priority to daily life activities rather than preventive health behavior. Another possible explanation for elderly people is that someone without daily exercise may not be able to maintain the Activity of Daily Living (ADL) for going to the health check venues. Our study suggests that persons who took positive coping behavior were more likely to engage in preventive health behavior for particular sex and age groups.

The same German study showed non-smoker people, both men and women, are likely to attend health checks. Our study showed similar results. In addition, our study also showed that elderly male ex-smoker and middle-aged female non-smoker were likely to attend. Therefore, non- or ex-smoker is associated with health check attendance in these sex- and age-groups. Our study suggests that negative coping behavior was related to avoidance from preventive health behavior in particular sex and age groups.

The German study and a preceding study in Austria [19] showed decreased health check attendance rate was associated with poor self-rated health. In the present study, the association between decreased health check attendance rate and poor self-rated health status was observed in women aged 65 years or older and bivariate analysis. Further research is required.

We observed the highest $\mathrm{OR}$ in young workingmen $(\mathrm{OR}=6.54)$. In this group, social status may have especially strong effect on the decision of attend health checks. The second highest OR was seen in young men with high physical activity $(\mathrm{OR}=4.00)$. Our study suggests that positive coping behavior in daily life is strongly associated with preventive health behavior in this group. Young person represent the majority of the active and productive population. Therefore, aggressive outreach programs may be required for non-attending men in this age group.

It is difficult to compare our study with previous studies because health care systems and their related health checks are different depending on the country. Nevertheless, our results were mostly consistent with previous studies analyzed by sex. In addition, we clarified the features of the relationship of social status and health-related behaviors with health check attendance by sex and age.

\subsection{Strengths and Limitations of This Study}

The strengths of this study include the use of data from a large sample of all residents in model areas and the high response rate. By using high response rate data, we excluded the self-selection bias due to under-representation of certain population groups in the sample as much as possible. Another strength is the clarification of health check attendance by the analysis of eligible persons across all ages stratified by age groups. Such an analysis on all ages has rarely been done up to now.

Nevertheless, there are several limitations to our study. One limitation arises 
from the measurement of variables considered in the study. The assessment of physical activity, vegetable consumption, alcohol consumption, and their definitions are different from previous studies, so we cannot compare with them exactly. Results were based on descriptive and self-reported data rather than administrative data. There was no validation done. Self-reported data might be influenced by mood or timing. Another limitation comes from using self-reported attendance at health checks as outcome measures. Self-reports on the use of health checks may be biased by confusing other medical services with health checks. In addition, because of the cross-sectional nature of the study, no causal relationships could be established and there may have been potential bias related to reverse causality. If respondents who had attended health checks had more favorable health-related behaviors as the result of their attendance, the effects of health-related behaviors on attendance may have been overestimated. Furthermore, we didn't have enough information of participants on socioeconomic status, education, marital status, and access to medical institutions. Studies without consideration of these variables may cause a bias.

\subsection{For Future Research}

Our findings suggest aggressive outreach programs may be required for young men. How to engage vulnerable groups in prevention services is an important issue for future research. These results suggest that health checks could waste valuable resources. One option is to adopt targeted health checks. Targeting limited resources to increase understanding in high-risk populations may be more cost-effective and increase the population benefits [26]. Several studies have demonstrated that targeted screening approaches may be more cost-effective than mass screening [32] [33]. Another option is to use resources in population-wide strategies to increase physical activity or to reduce smoking [26].

In this study, we didn't use some social variables above despite their importance. We should include these variables for future study.

Future research should examine possible reasons behind the differences in attendance by sex or age. It is also necessary to study the effects of health checks.

\subsection{Conclusion}

From these results, it can be concluded that the relationship of social status or health-related behaviors with health check attendance rates differs depending on sex and age groups. On the whole, the findings of this study suggest that population groups with increased risks of adverse health effects are less likely to attend health checks than those with decreased risks in particular sex and age groups. It indicates that diverse approaches are required to realize the full benefit of health checks. An increased participation of vulnerable groups might potentially contribute to achieving a positive effect on population health. The effective use of preventive services is a key factor in the reduction of important health concerns. It has the potential to make people healthier as well as to prolong their lives. 


\section{Acknowledgements}

The Kanazawa Study 2011 was commissioned by Kanazawa city. We would like to thank the study participants who responded to the survey and all of our colleagues of the department.

\section{References}

[1] Shippee, N.D., Mullan, R.J., Nabhan, M., Kermott, C.A., Hagen, P.T., Rhodes, D.J., Montori, V.M. and Murad, M.H. (2012) Adherence to Preventive Recommendations: Experience of a Cohort Presenting for Executive Health Care. Population Health Management, 15, 65-70. https://doi.org/10.1089/pop.2011.0029

[2] Ministry of Health, Labour and Welfare (2017) Data of the Specific Health Checkups and Specific Counseling.

http://www.mhlw.go.jp/bunya/shakaihosho/iryouseido01/info02a-2.html

[3] Ministry of Health, Labour and Welfare (2017) Health and Medical Services. http://www.mhlw.go.jp/english/wp/wp-hw6/dl/02e.pdf

[4] Ministry of Health, Labour and Welfare (2017) Specific Health Checkups and Specific Health Counseling. http://www.mhlw.go.jp/english/wp/wp-hw3/dl/2-007.pdf

[5] Culica, D., Rohrer, J., Ward, M., Hilsenrath, P. and Pomrehn, P. (2002) Medical Checkups: Who Does Not Get Them? American Journal of Public Health, 92, 88-91. https://doi.org/10.2105/AJPH.92.1.88

[6] Waller, D., Agass, M., Mant, D., Coulter, A., Fuller, A., et al. (1990) Health Checks in General Practice: Another Example of Inverse Care? British Medical Journal, 300, 1115-1118. https://doi.org/10.1136/bmj.300.6732.1115

[7] Wall, M. and Teeland, L. (2004) Non-Participants in a Preventive Health Examination for Cardiovascular Disease: Characteristics, Reasons for Non-Participation, and Willingness to Participate in the Future. Scandinavian Journal of Primary Health Care, 22, 248-251. https://doi.org/10.1080/02813430410006611

[8] Hsu, H. (2001) The Relationships between Health Beliefs and Utilization of Free Health Examinations in Older People Living in a Community Setting in Taiwan. Journal of Advanced Nursing, 35, 864-873. https://doi.org/10.1046/j.1365-2648.2001.01924.x

[9] Jones, A., Cronin, P.A. and Bowen, M. (1992) Comparison of Risk Factors for Coronary Heart Disease among Attenders and Non-Attenders at a Screening Programme. British Journal of General Practice, 43, 375-377.

[10] City, C. (1989) Influence of Fatness, Intelligence, Education and Sociodemographic Factors on Response Rate in a Health. Journal of Epidemiology and Community Health, 43, 369-374. https://doi.org/10.1136/jech.43.4.369

[11] Walker, M., Shaper, A.G. and Cook, D.G. (1987) Non-Participation and Mortality in a Prospective Study of Cardiovascular Disease. Journal of Epidemiology and Community Health, 41, 295-299. https://doi.org/10.1136/jech.41.4.295

[12] Thorogood, M., Coulter, A., Jones, L., Yudkin, P., Muir, J. and Mant, D. (1993) Factors Affecting Response to an Invitation to Attend for a Health Check. Journal of Epidemiology and Community Health, 47, 224-228. https://doi.org/10.1136/jech.47.3.224

[13] Griffiths, C., Cooke, S. and Toon, P. (1994) Registration Health Checks: Inverse Care in the Inner City? British Journal of General Practice, 44, 201-204. 
[14] Boshuizen, H.C., Viet, A.L., Picavet, H.S.J. and Botterweck, A. (2006) Non-Response in a Survey of Cardiovascular Risk Factors in the Dutch Population: Determinants and Resulting Biases. Public Health, 120, 297-308.

[15] French, J., Harding, K. and Stott, N. (1988) Invitation to Attend a Health Check in a General Practice Setting: Comparison of Attenders and Non-Attenders. Journal of the Royal College of General Practitioners, 38, 53-56.

[16] Christensen, B. and Christensen, B. (2009) Characteristics of Attenders and NonAttenders at Health Examinations for Ischaemic Heart Disease in General Practice Characteristics of Attenders and Non-Attenders at Health Examinations for Ischaemic Heart Disease in General Practice. Scandinavian Journal of Primary Health Care, 13, 26-31. https://doi.org/10.3109/02813439508996731

[17] Difford, F., Fornear, E. and Reading, C.A. (1987) Continuous Opportunistic and Systematic Screening for Hypertension with Computer Help: Analysis of NonResponders. British Medical Journal, 294, 1130-1132. https://doi.org/10.1136/bmj.294.6580.1130

[18] Hoebel, J., Starker, A., Jordan, S., Richter, M. and Lampert, T. (2014) Determinants of Health Check Attendance in Adults: Findings from the Cross-Sectional German Health Update (GEDA) Study. BMC Public Health, 14, 913. https://doi.org/10.1186/1471-2458-14-913

[19] Brunner-Ziegler, S., Rieder, A., Stein, K.V., Koppensteiner, R., Hoffmann, K. and Dorner, T.E. (2013) Predictors of Participation in Preventive Health Examinations in Austria. BMC Public Health, 13, 1138. https://doi.org/10.1186/1471-2458-13-1138

[20] Blair, S.N. and Brodney, S. (1999) Effects of Physical Inactivity and Obesity on Morbidity and Mortality: Current Evidence and Research Issues. Medicine and Science in Sports and Exercise, 31, 646-662. https://doi.org/10.1097/00005768-199911001-00025

[21] Hayashi, T., Tsumura, K., Suematsu, C., Okada, K., Fujii, S. and Endo, G. (1999) Walking to Work and the Risk for Hypertension in Men: The Osaka Health Survey. Annals of Internal Medicine, 131, 21-26. https://doi.org/10.7326/0003-4819-131-1-199907060-00005

[22] Hu, F.B., Manson, J.E., Stampfer, M.J., Colditz, G., Liu, S., Solomon, C.G. and Willett, W.C. (2001) Diet, Lifestyle, and the Risk of Type 2 Diabetes Mellitus in Women. The New England Journal of Medicine, 345, 790-797. https://doi.org/10.1056/NEJMoa010492

[23] Force, J.E.S.T. (2001) Established and Emerging Cardiovascular Risk Factors. American Heart Journal, 141, 49-57. https://doi.org/10.1067/mhj.2001.109951

[24] Greenlund, K.J., Giles, W.H., Keenan, N.L., Croft, J.B. and Mensah, G.A. (2002) Physician Advice, Patient Actions, and Health-Related Quality of Life in Secondary Prevention of Stroke through Diet and Exercise. Stroke, 33, 565-571. https://doi.org/10.1161/hs0202.102882

[25] Knowler, W.C., Barrett-Connor, E., Fowler, S.E., Hamman, R.F., Lachin, J.M., Walker, E.A. and Nathan, D.M. (2002) Reduction in the Incidence of Type 2 Diabetes with Lifestyle Intervention or Metformin. The New England Journal of Medicine, 346, 393-403. https://doi.org/10.1056/NEJMoa012512

[26] Dalton, A.R.H., Bottle, A., Okoro, C., Majeed, A. and Millett, C. (2011) Understanding of the NHS Health Checks Programme in a Deprived, Culturally Diverse Setting: Cross-Sectional Study. Journal of Public Health, 33, 422-429.

[27] Dryden, R., Williams, B., McCowan, C. and Themessl-Huber, M. (2012) What Do 
We Know about Who Does and Does not Attend General Health Checks? Findings from a Narrative Scoping Review. BMC Public Health, 12, 1. https://doi.org/10.1186/1471-2458-12-723

[28] World Health Organization. Obesity and Overweight. http://www.who.int/mediacentre/factsheets/fs311/en/

[29] Goldberg, D.P. and Hillier, V.F. (1979) A Scaled Version of the General Health Questionnaire. Psychological Medicine, 9, 139-145. https://doi.org/10.1017/S0033291700021644

[30] Sánchez-López, M.D.P. and Dresch, V. (2008) The 12-Item General Health Questionnaire (GHQ-12): Reliability, External Validity and Factor Structure in the Spanish Population. Psicothema, 20, 839-843.

[31] Pan, P.C. and Goldberg, D.P. (1990) A Comparison of the Validity of GHQ-12 and CHQ-12 in Chinese Primary Care Patients in Manchester. Psychological Medicine, 20, 931-940. https://doi.org/10.1017/S003329170003662X

[32] Chamnan, P., Simmons, R.K., Khaw, K.T., Wareham, N.J. and Griffin, S.J. (2010) Estimating the Population Impact of Screening Strategies for Identifying and Treating People at High Risk of Cardiovascular Disease: Modelling Study. BMJ, 340, c1693. https://doi.org/10.1136/bmj.c1693

[33] Lawson, K.D., Fenwick, E.A.L., Pell, A.C.H. and Pell, J.P. (2010) Comparison of Mass and Targeted Screening Strategies for Cardiovascular Risk: Simulation of the Effectiveness, Cost-Effectiveness and Coverage Using a Cross-Sectional Survey of 3921 People. Heart, 96, 208-212. https://doi.org/10.1136/hrt.2009.177204

\section{Submit or recommend next manuscript to SCIRP and we will provide best service for you:}

Accepting pre-submission inquiries through Email, Facebook, LinkedIn, Twitter, etc. A wide selection of journals (inclusive of 9 subjects, more than 200 journals)

Providing 24-hour high-quality service

User-friendly online submission system

Fair and swift peer-review system

Efficient typesetting and proofreading procedure

Display of the result of downloads and visits, as well as the number of cited articles

Maximum dissemination of your research work

Submit your manuscript at: http://papersubmission.scirp.org/

Or contact health@scirp.org 